\title{
WEAK ENDPOINT BOUNDS FOR MATRIX WEIGHTS
}

\author{
DAVID CRUZ-URIBE, OFS, JOSHUA ISRALOWITZ, KABE MOEN, SANDRA POTT, \\ AND ISRAEL P. RIVERA-RÍOS
}

\begin{abstract}
We prove quantitative matrix weighted endpoint estimates for the matrix weighted Hardy-Littlewood maximal operator, Calderón-Zygmund operators, and commutators of CZOs with scalar BMO functions, when the matrix weight is in the class $\mathrm{A}_{1}$ introduced by M. Frazier and S. Roudenko.
\end{abstract}

\section{INTRODUCTION}

In this paper we consider weak-type endpoint estimates for operators with matrix weights. In order to put our results into context, we first review briefly the scalar case. It is well-known that the Hardy-Littlewood maximal operator and all CalderónZygmund operators (CZOs) are bounded on $L^{p}(w)$ when $p>1$ and $w \in A_{p}$ : that is,

$$
[w]_{A_{p}}=\sup _{Q}\left(f_{Q} w(x) d x\right)\left(f_{Q} w(x)^{1-p^{\prime}} d x\right)^{p-1}<\infty,
$$

where the supremum is taken over all cubes $Q$ with sides parallel to the coordinate axes. These operators are not bounded on $L^{1}(w)$, but do map $L^{1}(w)$ into $L^{1, \infty}(w)$ when $w \in A_{1}$ : that is, for every cube $Q$ and almost every $x \in Q$,

$$
f_{Q} w(y) d y \leq[w]_{A_{1}} w(x)
$$

here $[w]_{A_{1}}$ is the infimum of all constants such that this inequality holds.

However, there is another version of the endpoint inequality. Given a weight $w$ and an operator $T$, for $1<p<\infty$, define $T_{w} f=w^{-\frac{1}{p}} T\left(w^{\frac{1}{p}} f\right)$. Then it is immediate that strong-type inequalities for $T_{w}$ are equivalent to weighted strong-type estimates for $T: T_{w}: L^{p}\left(\mathbb{R}^{d}\right) \rightarrow L^{p}\left(\mathbb{R}^{d}\right)$ if and only if $T: L^{p}(w) \rightarrow L^{p}(w)$. Consequently, we have that if $T$ is a CZO or the Hardy-Littlewood maximal operator, and if $w \in A_{p}$,

2010 Mathematics Subject Classification. Primary 42B20, 42B25, $42 \mathrm{~B} 35$.

Key words and phrases. Matrix weights, matrix $A_{p}$, maximal operators, Calderón-Zygmund operators, commutators, sparse operators.

Cruz-Uribe is supported by research funds from the Dean of the College of Arts \& Sciences, the University of Alabama; Isralowitz and Moen are supported by the Simons Foundation; Rivera-Ríos is supported by grant PIP (CONICET) 11220130100329CO. 
then $T_{w}: L^{p}\left(\mathbb{R}^{d}\right) \rightarrow L^{p, \infty}\left(\mathbb{R}^{d}\right)$. This suggests that when $p=1$ and $w \in A_{1}$, we should have weak-type inequalities of the form

$$
\left|\left\{x: w(x) T\left(f w^{-1}\right)(x)>\lambda\right\}\right| \lesssim \frac{1}{\lambda} \int_{\mathbb{R}^{d}}|f(x)| d x .
$$

Muckenhoupt and Wheeden [21] first proved such inequalities when $d=1$ for the Hardy-Littlewood maximal operator and the Hilbert transform; their results were extended to higher dimensions and arbitrary CZOs (as well as the maximal operator) in [5]. These estimates are much more delicate and even for the maximal operator are much more difficult to prove than the more standard endpoint result considered above. Moreover, it was shown in [21] that the $A_{1}$ condition is not necessary even for the maximal operator; they showed, for instance, that this endpoint inequality holds when $w(x)=|x|^{-1}$.

Remark. There has been a great deal of interest in generalizing these results to the two-weight setting; the original motivation (even in the one-weight setting) is that such inequalities arise naturally in the theory of interpolation with change of measure of Stein and Weiss [27]. See [1] for a short history.

We now consider matrix weights: our goal is to generalize (1.1) to this setting. To state our results we first give some basic definitions. For more details, see [6, 10, 26, A matrix weight $W$ is an $n \times n$ self-adjoint matrix function with locally integrable entries such that $W(x)$ is positive definite for a.e. $x \in \mathbb{R}^{d}$. Define $W^{r}$ for any $r \in \mathbb{R}$ via diagonalization. Define the operator norm of $W(x)$ by

$$
|W(x)|_{\text {op }}=\sup _{\substack{e \in \mathbb{C}^{n} \\|e|=1}}|W(x) e| .
$$

Finally, for all $1 \leq p<\infty$, define $L^{p}(W)$ to be the collection of measurable, vectorvalued functions $f: \mathbb{R}^{d} \rightarrow \mathbb{C}^{n}$ such that

$$
\|f\|_{L^{p}(W)}=\left(\int_{\mathbb{R}^{d}}\left|W(x)^{\frac{1}{p}} f(x)\right|^{p} d x\right)^{\frac{1}{p}}<\infty .
$$

Given a linear operator $T, 1 \leq p<\infty$, and a matrix weight $W$, define the matrix operator $T_{W}$ by

$$
T_{W} f(x)=W(x)^{\frac{1}{p}} T\left(W^{-\frac{1}{p}} f\right)(x) ;
$$

as before, $T_{W}: L^{p}\left(\mathbb{R}^{d}, \mathbb{C}^{n}\right) \rightarrow L^{p}\left(\mathbb{R}^{d}, \mathbb{C}^{n}\right)$ if and only if $T: L^{p}(W) \rightarrow L^{p}(W)$. It was shown by Christ and Goldberg [2,10] that for $p>1$, if $T$ is a CZO, then this inequality holds if and only if $W$ satisfies the matrix $\mathrm{A}_{p}$ condition,

$$
[W]_{\mathrm{A}_{p}}=\sup _{Q} f_{Q}\left(f_{Q}\left|W(x)^{\frac{1}{p}} W(y)^{-\frac{1}{p}}\right|_{\mathrm{op}}^{p^{\prime}} d y\right)^{\frac{p}{p^{\prime}}} d x<\infty .
$$

Note that this formulation of the matrix $\mathrm{A}_{p}$ condition is due to Roudenko [26]; see this paper or [2,10] for the earlier, equivalent definition in terms of norms. While the 
maximal operator is not linear, they showed that a variant of the maximal operator (now referred to as the Christ-Goldberg maximal operator,

$$
M_{W} f(x)=\sup _{Q \ni x} f_{Q}\left|W(x)^{\frac{1}{p}} W^{-\frac{1}{p}}(y) f(y)\right| d y
$$

is bounded on $L^{p}\left(\mathbb{R}^{d}, \mathbb{C}^{n}\right)$ if and only if $W \in \mathrm{A}_{p}, 1<p<\infty$.

In the setting of matrix weights, it is unclear how to define the weak-type space $L^{p, \infty}(W)$; therefore, it is natural to use the strong-type bounds to get unweighted, weak $(p, p)$ bounds for $T_{W}$ or $M_{W}$. Here, we argue directly to prove weak $(1,1)$ estimates for $T_{W}$ when $T$ is a CZO, and for the Christ-Goldberg maximal operator $M_{W}$. The appropriate weight class is matrix $A_{1}$, first defined by Frazier and Roudenko [7]: a matrix weight $W$ belongs to $\mathrm{A}_{1}$ if

$$
[W]_{\mathrm{A}_{1}}=\operatorname{ess} \sup _{x \in \mathbb{R}^{d}} \sup _{Q \ni x} f_{Q}\left|W(y) W^{-1}(x)\right|_{\mathrm{op}} d y<\infty .
$$

Note that in the scalar case, when $n=1$, this definition reduces immediately to the definition of scalar $A_{1}$. If $W \in \mathrm{A}_{1}$, then we have that $|W(\cdot)|_{\text {op }}$ is in scalar $A_{1}$; see [6, Lemma 4.4]. Therefore, we can define the scalar $A_{\infty}$ constant of $W$ by

$$
[W]_{\mathrm{A}_{\infty}^{\mathrm{sc}}}=\sup _{e \in \mathbb{C}^{n}}[|W(\cdot) e|]_{\mathrm{A}_{\infty}} .
$$

This constant was first introduced in the $p=2$ setting in [22] and for $1<p<\infty$ in 3]. In the theory of scalar weights there are several equivalent definitions of $A_{\infty}$; we will use the sharp, Fujii-Wilson definition. The precise definition does not directly matter as we will use this condition indirectly; see [12,13] for details.

We can now state our first two results.

Theorem 1.1. Define $M_{W}$ by (1.3) with $p=1$. Given $W \in A_{1}$, then for all $f \in$ $L^{1}\left(\mathbb{R}^{d}, \mathbb{C}^{n}\right)$ and $\lambda>0$,

$$
\left|\left\{x \in \mathbb{R}^{d}: M_{W} f(x)>\lambda\right\}\right| \lesssim \frac{[W]_{A_{1}}[W]_{A_{\infty}^{s c}}}{\lambda} \int_{\mathbb{R}^{d}}|f(x)| d x .
$$

Theorem 1.2. Define $T_{W}$ by (1.2) with $p=1$. Given $W \in A_{1}$ then for all $f \in$ $L^{1}\left(\mathbb{R}^{d}, \mathbb{C}^{n}\right)$ and $\lambda>0$,

$$
\left|\left\{x \in \mathbb{R}^{d}:\left|T_{W} f(x)\right|>\lambda\right\}\right| \lesssim \frac{[W]_{A_{1}}[W]_{A_{\infty}^{s c}}}{\lambda} \int_{\mathbb{R}^{d}}|f(x)| d x .
$$

It follows from the definition that $[W]_{\mathrm{A}_{\infty}^{\mathrm{sc}}} \lesssim[W]_{\mathrm{A}_{1}}$, so in both of these results we can estimate the constant by $[W]_{\mathrm{A}_{1}}^{2}$. While we are able to give a quantitative estimate in terms of the $\mathrm{A}_{1}$ constant, we do not believe that either result is sharp. In the scalar case, for the Hardy-Littlewood maximal operator it is well known (though not explicitly in the literature) that the sharp constant is $[w]_{A_{1}}$. For CZOs the sharp constant is $[w]_{A_{1}}\left(1+\log \left([w]_{A_{1}}\right)\right)$ : see Lerner, Ombrosi and Pérez [19] for the upper bound and [18] for the lower bound. 
Remark. When $1<p<\infty$, direct estimates for the best constant in the weak $(p, p)$ inequalities for these operators are not known. From the strong $(p, p)$ inequalities, we have that an upper bound on the constant for $M_{W}$ is $[W]_{\mathrm{A}_{p}}^{\frac{p^{\prime}}{p}}$ (see [14]) and for $T_{W}$ is $[W]_{\mathrm{A}_{p}}^{1+\frac{1}{p-1}-\frac{1}{p}}($ see [3] $)$. It is an open question whether our techniques can be used to prove better weak type estimates.

Remark. As we noted above, even in the scalar case the $A_{1}$ condition is not necessary for $M_{W}$ to satisfy the weak $(1,1)$ inequality. However, $\mathrm{A}_{1}$ weights are characterized by a weak $(1,1)$ inequality for a closely related, "auxiliary" maximal operator $M_{W}^{\prime}$, that was introduced by Christ and Goldberg [2,10] and which plays an important role in studying $M_{W}$. See [3, Theorem 1.21] where this is proved in a more general context.

Finally, we can also use our techniques to prove a quantitative, weak-type estimate for commutators of CZOs. Let $T$ be a CZO and let $b \in B M O$. Define the commutator $[T, b] f(x)=T(b f)(x)-b(x) T f(x)$, and define the matrix weighted commutator $C_{b, W}(T) f=W[T, b] W^{-1} f$. Even in the scalar case commutators are more singular and do not satisfy weak $(1,1)$ bounds. Rather, the natural endpoint condition involves an $L \log L$ estimate: see Pérez and Pradolini [24,25]. Let $\Phi(t)=t \log (e+t)$; then we have the following result.

Theorem 1.3. Given $W \in A_{1}$, then for all $f \in L^{1}\left(\mathbb{R}^{d}, \mathbb{C}^{n}\right)$ and $\lambda>0$,

$$
\begin{aligned}
& \left|\left\{x \in \mathbb{R}^{d}:\left|C_{b, W}(T) f(x)\right|>\lambda\right\}\right| \\
& \quad \lesssim\|b\|_{B M O}[W]_{A_{1}} \max \left\{\log \left([W]_{A_{1}}+e\right),[W]_{A_{\infty}^{s c}}\right\}^{2} \int_{\mathbb{R}^{d}} \Phi\left(\frac{|f(x)|}{\lambda}\right) d x .
\end{aligned}
$$

The remainder of this paper is organized as follows. In Section 2 we provide some preliminary results about matrix weights and about domination via sparse operators. In Section 3 we prove Theorems 1.1 and 1.2. Finally, in Section 4 we prove Theorem 1.3 .

Throughout, $d$ will denote the dimension of the underlying space $\mathbb{R}^{d}$, and $n$ will be dimension of space $\mathbb{C}^{n}$ in which vector-valued functions take their range. All matrices will be $n \times n$ matrices. If we write $A \lesssim B$, then there exists a constant $c$ such that $A \leq c B$. By $A \equiv B$ we mean $A \lesssim B$ and $B \lesssim A$. The implicit constants might depend on $n$, $d$, or the given CZO, but will not depend on the matrix weight $W$.

\section{Preliminaries}

In this section we gather a few additional facts about matrix weights and also give the results on sparse domination which are central to our proofs. 
First, as we noted above, if $W \in \mathrm{A}_{1}$, then we have that $|W(\cdot)|_{\text {op }}$ is a scalar $A_{1}$ weight [6, Lemma 4.4]. Moreover, we in fact have that for any $e \in \mathbb{C}^{n},|W(\cdot) e| \in A_{1}$, and

$$
[W]_{\mathrm{A}_{1}}=\sup _{e \in \mathbb{C}^{n}}[|W(\cdot) e|]_{A_{1}}
$$

Central to estimating matrix weighted operators is the concept of a reducing matrix. These were first introduced in [2,10] when $p>1$, and when $p=1$ in [7]. Given a norm $\rho$ on $\mathbb{C}^{n}$, it is well known (see [23, Lemma 11.4] for a self contained and simple proof) that there exists a positive definite $n \times n$ matrix $A$ such that for any $e \in \mathbb{C}^{n}$ we have $|A e| \approx \rho(e)$. We refer to this matrix as a reducing matrix of $\rho$. (Note that the matrix $A$ is not unique, but this is not important in practice.) In particular, given a matrix weight $W$ and any measurable $Q \subseteq \mathbb{R}^{d}$ with $0<|Q|<\infty$, we have that $e \mapsto f_{Q}|W(y) e| d y$ is a norm on $\mathbb{C}^{n}$. Hereafter we will denote by $\mathcal{W}_{Q}$ any reducing matrix for this norm, so that

$$
\left|\mathcal{W}_{Q} e\right| \approx f_{Q}|W(y) e| d y .
$$

We can also define the matrix $\mathrm{A}_{1}$ condition in terms of reducing matrices. Given a cube $Q$ and $x \in Q$, we have that if $\left\{e_{j}\right\}_{j}$ is the standard basis for $\mathbb{C}^{n}$, then

$$
\begin{aligned}
& \left|\mathcal{W}_{Q} W^{-1}(x)\right|_{\mathrm{op}} \approx \sum_{j=1}^{n}\left|\mathcal{W}_{Q} W^{-1}(x) e_{j}\right| \\
& \quad \approx \sum_{j=1}^{n} f_{Q}\left|W(y) W^{-1}(x) e_{j}\right| d y \approx f_{Q}\left|W(y) W^{-1}(x)\right|_{\mathrm{op}} d y .
\end{aligned}
$$

Hence,

$$
[W]_{A_{1}} \approx \sup _{Q} \sup _{x \in Q}\left|\mathcal{W}_{Q} W^{-1}(x)\right|_{\mathrm{op}}
$$

To prove our results, we will show that we can reduce each weak-type estimate to proving an analogous result for a so-called sparse operator. To define these operators first we recall the machinery of general dyadic grids as defined in [17]; we refer the reader there for complete details. We will need the fact that every cube in $\mathbb{R}^{d}$ can be approximated by a dyadic cube from one of finitely many dyadic grids (see the corollary of [17, Theorem 3.1]).

Lemma 2.1. There exist dyadic grids $\mathcal{D}^{1}, \ldots, \mathcal{D}^{3^{d}}$ such that given any cube $Q$ there exists $1 \leq \alpha \leq 3^{d}$ and $Q^{\alpha} \in \mathcal{D}^{\alpha}$ such that $Q \subset Q^{\alpha}$ and $\ell\left(Q^{\alpha}\right) \leq 6 \ell(Q)$.

Given $\eta \in(0,1)$ we say that $\mathcal{S} \subset \mathcal{D}$ is a $\eta$-sparse family if for every $Q \in \mathcal{S}$ there exists a measurable subset $E_{Q} \subset Q$ such that

(1) $\eta|Q| \leq\left|E_{Q}\right|$.

(2) The sets $E_{Q}$ are pairwise disjoint. 
Further, given $\Lambda>1$ we say that $\mathcal{S} \subset \mathcal{D}$ is a $\Lambda$ Carleson family if for every $Q \in \mathcal{S}$,

$$
\sum_{P \in \mathcal{S}, P \subseteq Q}|P| \leq \Lambda|Q| \text {. }
$$

Clearly every $\eta$-sparse family is $\eta^{-1}$ Carleson, since

$$
\sum_{P \in \mathcal{S}, P \subseteq Q}|P| \leq \eta^{-1} \sum_{P \in \mathcal{S}, P \subseteq Q}\left|E_{P}\right| \leq \Lambda^{-1}|Q| .
$$

Though less obvious, the converse is true:. every $\Lambda$ Carleson family is $\Lambda^{-1}$ sparse [17, Lemma 6.3]. We will also use without further comment the fact that every $\Lambda$ Carleson family can be written as a union of $m$ Carleson families, each of which is $1+\frac{\Lambda-1}{m}$ Carleson [17, Lemma 6.6]. Hereafter we will sometimes refer to a family as sparse or Carleson without reference to $\eta$ or $\Lambda$ if the specific values of these constants are unimportant.

To estimate CZOs applied to vector-valued functions, we will use the convex body domination that was introduced by F. Nazarov, S. Petermichl and A. Volberg [22]. Given a cube $Q$ and a function $f \in L^{1}\left(Q, \mathbb{C}^{n}\right)$, define

$$
\langle\langle f\rangle\rangle_{Q}=\left\{f_{Q} f \varphi d x: \varphi \in B_{L^{\infty}}(Q)\right\},
$$

where $B_{L^{\infty}(Q)}=\left\{\phi \in L^{\infty}(Q, \mathbb{R}):\|\phi\|_{L^{\infty}} \leq 1\right\}$. They proved that $\left\langle\langle f\rangle_{Q}\right.$ is a symmetric, convex and compact set in $\mathbb{C}^{n}$.

The following result was first proved in [22]; we give it here in the version found in [11, Corollary 2.3.18]. To state it, recall that given a linear operator $T$, the grandmaximal operator $M_{T}$, defined by A. Lerner [16], is

$$
M_{T} f(x)=\sup _{Q \ni x} \sup _{y \in Q}\left|T\left(f \chi_{\mathbb{R}^{n} \backslash 3 Q}\right)(y)\right| .
$$

Theorem 2.2. Let $T: L^{1}\left(\mathbb{R}^{d}\right) \rightarrow L^{1, \infty}\left(\mathbb{R}^{d}\right)$ be a linear operator such that $M_{T}$ : $L^{1}\left(\mathbb{R}^{d}\right) \rightarrow L^{1, \infty}\left(\mathbb{R}^{d}\right)$. For $f \in L_{c}^{1}\left(\mathbb{R}^{d} ; \mathbb{C}^{n}\right)$ and $\varepsilon \in(0,1)$, there exist $3^{d}, 3^{-d}(1-\varepsilon)$ sparse collections of dyadic cubes (drawn from the dyadic grids in Lemma 2.1) such that

$$
T f(x) \in \frac{c_{d, n} c_{T}}{\varepsilon} \sum_{j=1}^{3^{d}} \sum_{Q \in \mathcal{S}_{j}}\left\langle\langle f\rangle_{Q} \chi_{Q}(x)\right.
$$

where $c_{T}=\|T\|_{L^{1} \rightarrow L^{1, \infty}}+\left\|M_{T}\right\|_{L^{1} \rightarrow L^{1, \infty}}$. In particular, there exist functions $k_{Q} \in$ $B_{L^{\infty}(Q \times Q)}$ such that

$$
T f(x)=\frac{c_{d, n} c_{T}}{\varepsilon} \sum_{j=1}^{3^{d}} \sum_{Q \in \mathcal{S}_{j}}\left(f_{Q} k_{Q}(x, y) f(y) d y\right) \chi_{Q}(x) .
$$

Lerner proved that for Calderón-Zygmund operators,

$$
\left\|M_{T}\right\|_{L^{1} \rightarrow L^{1, \infty}} \leq c_{d}\left(\|T\|_{L^{2} \rightarrow L^{2}}+c_{K}+\|\omega\|_{\text {Dini }}\right) .
$$


See [16] for the precise definitions of the Dini condition and the Dini "norm" of the kernel $K$ of a CZO $T$. It is also known that

$$
\|T\|_{L^{1} \rightarrow L^{1, \infty}} \leq c_{d}\left(\|T\|_{L^{2} \rightarrow L^{2}}+\|\omega\|_{\text {Dini }}\right) .
$$

Consequently Theorem 2.2 holds for a Calderón-Zygmund operator $T$ with

$$
c_{T}=\|T\|_{L^{2} \rightarrow L^{2}}+c_{K}+\|\omega\|_{\text {Dini }}
$$

The sparse convex body domination can also be extended to commutators. In fact, somewhat surprisingly, we can use a " $2 \times 2$ block matrix trick" inspired by [9] in conjunction with Theorem 2.2 to obtain the corresponding result for commutators. In particular, the following was very recently proved in [15], extending [20, Theorem 1.1] (and in fact providing a very short proof of [20, Theorem 1.1].) For completeness we include the relatively short proof.

Theorem 2.3. Let $T: L^{1}\left(\mathbb{R}^{d}\right) \rightarrow L^{1, \infty}\left(\mathbb{R}^{d}\right)$ be a linear operator such that $M_{T}$ : $L^{1}\left(\mathbb{R}^{d}\right) \rightarrow L^{1, \infty}\left(\mathbb{R}^{d}\right)$. For $f \in L_{c}^{1}\left(\mathbb{R}^{d} ; \mathbb{C}^{n}\right)$ and every $b \in L_{\text {loc }}^{1}\left(\mathbb{R}^{d}\right)$ where bf $\in$ $L^{1}\left(\mathbb{R}^{d}, \mathbb{C}^{n}\right)$, and $\varepsilon \in(0,1)$ there exist there exist $3^{d}, 3^{-d}(1-\varepsilon)$-sparse collections of dyadic cubes (drawn from the dyadic grids in Lemma 2.1) such that

$$
[b, T] f(x) \in \frac{c_{d, n} c_{T}}{\varepsilon} \sum_{j=1}^{3^{d}} \sum_{Q \in \mathcal{S}_{j}}\left[( b ( x ) - b _ { Q } ) \left\langle\langle f\rangle_{Q} \chi_{Q}(x)+\left\langle\left\langle\left(b-b_{Q}\right) f\right\rangle_{Q} \chi_{Q}(x)\right]\right.\right.
$$

where each $C_{S}$ is a constant and $c_{T}=\|T\|_{L^{1} \rightarrow L^{1, \infty}}+\left\|M_{T}\right\|_{L^{1} \rightarrow L^{1, \infty}}$. In particular, there exist functions $k_{Q} \in B_{L^{\infty}(Q \times Q)}$ such that

$$
\begin{aligned}
{[b, T] f(x)=} & \frac{c_{d, n} c_{T}}{\varepsilon} \sum_{j=1}^{3^{d}} \sum_{Q \in \mathcal{S}_{j}}\left(b(x)-b_{Q}\right)\left(f_{Q} k_{Q}(x, y) f(y) d y\right) \chi_{Q}(x) \\
& -\frac{c_{d, n} c_{T}}{\varepsilon} \sum_{j=1}^{3^{d}} \sum_{Q \in \mathcal{S}_{j}}\left(f_{Q} k_{Q}(x, y)\left(b(y)-b_{Q}\right) f(y) d y\right) \chi_{Q}(x) .
\end{aligned}
$$

Proof. Let $f \in L_{c}^{\infty}\left(\mathbb{R}^{d}, \mathbb{C}^{n}\right)$. By Theorem 2.2 there exists sparse collections of cubes $\left\{\mathcal{S}_{j}\right\}_{j=1}^{3^{d}}$ and $k_{Q}(x, y)$ with $\left\|k_{Q}\right\|_{L^{\infty}\left(\mathbb{R}^{d} \times \mathbb{R}^{d}\right)}=1$ where

$$
T f(x)=c_{d, n} c_{T} \sum_{j=1}^{3^{d}} \sum_{Q \in \mathcal{S}_{j}}\left(f_{Q} k_{Q}(x, y) f(y) d y\right) \chi_{Q}(x) .
$$

Define the $\mathbb{C}^{2 n}$ valued function $\tilde{f}$ by

$$
\tilde{f}(x)=\left(\begin{array}{c}
f(x) \\
f(x)
\end{array}\right)
$$


and define the $2 n \times 2 n$ block matrix $\Phi(x)$ by

$$
\Phi(x)=\left(\begin{array}{cc}
1_{n \times n} & b(x) \otimes 1_{n \times n} \\
0 & 1_{n \times n}
\end{array}\right)
$$

so that

$$
\Phi^{-1}(x)=\left(\begin{array}{cc}
1_{n \times n} & -b(x) \otimes 1_{n \times n} \\
0 & 1_{n \times n}
\end{array}\right)
$$

Direct computation shows

$$
\Phi(x)\left(T \Phi^{-1} \tilde{f}\right)(x)=\left(\begin{array}{c}
T f(x)-[T, b] f(x) \\
T f(x)
\end{array}\right)
$$

and

$$
\Phi^{-1}(y) \tilde{f}(y)=\left(\begin{array}{cc}
1_{n \times n} & -b(y) \otimes 1_{n \times n} \\
0 & 1_{n \times n}
\end{array}\right)\left(\begin{array}{l}
f(y) \\
f(y)
\end{array}\right)=\left(\begin{array}{c}
f(y)-f(y) b(y) \\
f(y)
\end{array}\right)
$$

Since $\Phi^{-1} \tilde{f} \in L_{c}^{1}$, plugging $\Phi^{-1} \tilde{f}$ into (2.4) gives

$$
\begin{aligned}
\Phi(x)\left(T \Phi^{-1} \tilde{f}\right)(x) & =c_{d, n} c_{T} \sum_{j=1}^{3^{d}} \sum_{Q \in \mathcal{S}_{j}} \Phi(x)\left(\begin{array}{c}
\left\langle k_{Q}(x, \cdot)(f-f b)\right\rangle_{Q} \\
\left\langle k_{Q}(x, \cdot) f\right\rangle_{Q}
\end{array}\right) \chi_{Q}(x) \\
& =c_{d, n} c_{T} \sum_{j=1}^{3^{d}} \sum_{Q \in \mathcal{S}_{j}}\left(\begin{array}{c}
\left\langle k_{Q}(x, \cdot)(f-f b)\right\rangle_{Q}+b(x)\left\langle k_{Q}(x, \cdot) f\right\rangle_{Q} \\
\left\langle k_{Q}(x, \cdot) f\right\rangle_{Q}
\end{array}\right) \chi_{Q}(x) .
\end{aligned}
$$

However, adding and subtracting $\left\langle k_{Q}(x, \cdot) f\right\rangle_{Q}\langle b\rangle_{Q}$ to the first component, we get

$$
\begin{aligned}
& \Phi(x)\left(T \Phi^{-1} \tilde{f}\right)(x) \\
& =c_{d, n} c_{T} \sum_{j=1}^{3^{d}} \sum_{Q \in \mathcal{S}_{j}}\left(\begin{array}{c}
\left\langle k_{Q}(x, \cdot)(f-f b)\right\rangle_{Q}+b(x)\left\langle k_{Q}(x, \cdot) f\right\rangle_{Q} \\
\left\langle k_{Q}(x, \cdot) f\right\rangle_{Q}
\end{array}\right) \chi_{Q}(x) \\
& =c_{d, n} c_{T} \sum_{j=1}^{3^{d}} \sum_{Q \in \mathcal{S}_{j}}\left(\begin{array}{c}
\left\langle k_{Q}(x, \cdot) f\right\rangle_{Q}+\left\langle k_{Q}(x, \cdot) f\left(\langle b\rangle_{Q}-b\right)\right\rangle_{Q}+\left(b(x)-\langle b\rangle_{Q}\right)\left\langle k_{Q}(x, \cdot) f\right\rangle_{Q} \\
\left\langle k_{Q}(x, \cdot) f\right\rangle_{Q}
\end{array}\right) \chi_{Q}(x) .
\end{aligned}
$$

Thus,

$$
\begin{aligned}
{[T, b] f(x) } & =c_{d, n} c_{T} \sum_{j=1}^{3^{d}} \sum_{Q \in \mathcal{S}_{j}} f_{Q} k_{Q}(x, y)\left(b(y)-\langle b\rangle_{Q}\right) f(y) d y \\
& -\left(b(x)-\langle b\rangle_{Q}\right)\left(f_{Q} f(y) k_{Q}(x, y) f(y) d y .\right)
\end{aligned}
$$




\section{Proofs of Theorems 1.1 And 1.2}

We will first need to prove a sparse domination of the maximal function which is more useful for us than the linearization in [2,10]. Note that a similar stopping time argument was used in [15] to prove the sharp bound

$$
\left\|M_{W}\right\|_{L^{p} \rightarrow L^{p}} \lesssim[W]_{\mathrm{A}_{1}}^{\frac{1}{p}}
$$

when $1<p<\infty$. Given a dyadic grid $\mathcal{D}$, define

$$
M_{W}^{\mathcal{D}} f(x)=\sup _{\substack{Q \ni x \\ Q \in \mathcal{D}}} f_{Q}\left|W(x) W^{-1}(y) f(y)\right| d y
$$

and for a sparse collection $\mathcal{S}$ of dyadic cubes, let

$$
\mathcal{T}_{\mathcal{S}, W} f(x)=\sum_{Q \in \mathcal{S}}\left|W(x) \mathcal{W}_{Q}^{-1}\right|_{\mathrm{op}}\left\langle\left|\mathcal{W}_{Q} W^{-1} f\right|\right\rangle_{Q} \chi_{Q}(x)
$$

Lemma 3.1. Let $W$ be any matrix weight and $f \in L^{1}\left(\mathbb{R}^{d} ; \mathbb{C}^{n}\right)$ have compact support. Then there exists $3^{d}$ sparse collections $\mathcal{S}_{j}$ where

$$
M_{W} f(x) \lesssim \sum_{j=1}^{3^{d}} \mathcal{T}_{\mathcal{S}_{j}, W} f(x)
$$

Proof. By Lemma 2.1, it is enough to prove Lemma 3.1 for $M_{W}^{\mathcal{D}} f$ where $\mathcal{D}$ is a fixed dyadic grid. Furthermore, since $f$ has compact support, we can replace $M_{W}^{\mathcal{D}} f$ by $M_{W, J}^{\mathcal{D}}$ for some $J \in \mathcal{D}$, where

$$
M_{W, J}^{\mathcal{D}} f(x)=\sup _{\substack{Q \ni x \\ Q \in \mathcal{D}(J)}} f_{Q}\left|W(x) W^{-1}(y) f(y)\right| d y .
$$

Let $\mathscr{J}(J)$ denote the maximal cubes $L \in \mathcal{D}(J)$ (if any exist) where

$$
\left\langle\left|\mathcal{W}_{J} W^{-1} f\right|\right\rangle_{L}>2\left\langle\left|\mathcal{W}_{J} W^{-1} f\right|\right\rangle_{J} .
$$

By maximality we have

$$
\begin{aligned}
\sum_{L \in \mathscr{J}(J)}|L| & <\frac{1}{2\left\langle\left|\mathcal{W}_{J} W^{-1} f\right|\right\rangle_{J}} \sum_{L \in \mathscr{J}(J)} \int_{L}\left|\mathcal{W}_{J} W^{-1}(y) f(y)\right| d y \\
& \leq \frac{1}{2\left\langle\left|\mathcal{W}_{J} W^{-1} f\right|\right\rangle_{J}} \int_{J}\left|\mathcal{W}_{J} W^{-1}(y) f(y)\right| d y \\
& =\frac{|J|}{2}
\end{aligned}
$$


Now let $\mathcal{F}(J)$ be the collection of cubes in $\mathcal{D}(J)$ that are not a subset of any cube $I \in \mathscr{J}(J)$. We then have

$$
\begin{aligned}
\sup _{\substack{Q \ni x \\
Q \in \mathcal{D}(J)}} f_{Q}\left|W(x) W^{-1}(y) f(y)\right| d y \\
\leq \sup _{\substack{Q \ni x \\
Q \in \mathscr{F}(J)}} f_{Q}\left|W(x) W^{-1}(y) f(y) \chi_{\cup \mathscr{J}(J)}(y)\right| d y \\
\quad+\sup _{\substack{Q \ni x \\
Q \in \mathscr{F}(J)}} f_{Q}\left|W(x) W^{-1}(y) f(y) \chi_{J \backslash \cup \mathscr{J}(J)}(y)\right| d y \\
\quad+\sum_{L \in \mathscr{J}(J)} \sup _{\substack{Q \ni x \\
Q \in \mathcal{D}(L)}} f_{Q}\left|W(x) W^{-1}(y) f(y)\right| d y \\
:=A_{1}(x)+A_{2}(x)+\sum_{L \in \mathscr{J}(J)} \sup _{\substack{Q \ni x \\
Q \in \mathcal{D}(L)}} f_{Q}\left|W(x) W^{-1}(y) f(y)\right| d y .
\end{aligned}
$$

We first estimate $A_{1}(x)$. Let $x \in Q \in \mathscr{F}(J)$ and assume also $x \in I \in \mathscr{J}(J)$. Then by definition of $\mathscr{F}(J)$ we must have $I \subsetneq Q \subseteq J$ so that

$$
\begin{aligned}
A_{1}(x) & \leq \sup _{I \in \mathscr{J}(J)} \sup _{J \supseteq Q \supseteq I \ni x} f_{Q}\left|W(x) W^{-1}(y) f(y)\right| d y \\
& \leq\left|W(x) \mathcal{W}_{J}^{-1}\right|_{\text {op }} \sup _{I \in \mathscr{J}(J)} \sup _{J \supseteq Q \supseteq I} f_{Q}\left|\mathcal{W}_{J} W^{-1}(y) f(y)\right| d y \\
& \leq 2\left|W(x) \mathcal{W}_{J}^{-1}\right|_{\mathrm{op}}\left\langle\left|\mathcal{W}_{J} W^{-1} f\right|\right\rangle_{J} \chi_{J}(x)
\end{aligned}
$$

where the last inequality follows from maximality.

Next, to estimate $A_{2}(x)$, let $x \notin \cup_{L \in \mathscr{J}(J)} L$ and pick a sequence $L_{k}^{x}$ of nested dyadic cubes where

$$
\left\{L_{k}^{x}\right\}=\{L \in \mathscr{F}(J): x \in L\}=\{L \in \mathcal{D}(J): x \in L\} .
$$

But if

$$
\sup _{k}\left\langle\left|\mathcal{W}_{J} W^{-1} f\right|\right\rangle_{L_{k}^{x}}>2\left\langle\left|\mathcal{W}_{J} W^{-1} f\right|\right\rangle_{J}
$$

then for some $k$ we have

$$
\left\langle\left|\mathcal{W}_{J} W^{-1} f\right|\right\rangle_{L_{k}^{x}}>2\left\langle\left|\mathcal{W}_{J} W^{-1} f\right|\right\rangle_{J}
$$

which means that $x \in L_{k}^{x} \subseteq Q$ for some $Q \in \mathscr{J}(J)$. Thus,

$$
\begin{aligned}
A_{2}(x) & \leq \sup _{k} f_{L_{k}^{x}}\left|W(x) W^{-1}(y) f(y)\right| d y \\
& \left.\leq\left|W(x) \mathcal{W}_{J}^{-1}\right|_{\text {op }} \sup _{k}\langle| \mathcal{W}_{J} W^{-1} f\right)|\rangle_{L_{k}^{x}}
\end{aligned}
$$




$$
\leq 2\left|W(x) \mathcal{W}_{J}^{-1}\right|_{\mathrm{op}}\left\langle\left|\mathcal{W}_{J} W^{-1} f\right|\right\rangle_{J} \chi_{J}(x)
$$

Iteration now completes the proof

Proof of Theorems 1.1 and 1.2. By Fatou's lemma for weak type estimates, we may assume that $f$ has compact support. By Theorem 2.2 we have that there exists

$$
\begin{aligned}
\left|T_{W} f(x)\right| & \lesssim \sum_{j=1}^{3^{d}} \sum_{Q \in \mathcal{S}_{j}} f_{Q}\left|W(x) W^{-1}(y) f(y)\right| d y \cdot \chi_{Q}(x) \\
& \leq \sum_{j=1}^{3^{d}} \sum_{Q \in \mathcal{S}_{j}}\left|W(x) \mathcal{W}_{Q}^{-1}\right|_{\mathrm{op}} f_{Q}\left|\mathcal{W}_{Q} W^{-1}(y)\right|_{\mathrm{op}}|f(y)| d y \cdot \chi_{Q}(x) \\
& \leq[W]_{\mathrm{A}_{1}} \sum_{j=1}^{3^{d}} \sum_{Q \in \mathcal{S}}\left|W(x) \mathcal{W}_{Q}^{-1}\right|_{\mathrm{op}} f_{Q}|f(y)| d y \cdot \chi_{Q}(x) .
\end{aligned}
$$

Also note that Lemma 3.1 gives us the same estimate for $M_{W} f$. Therefore, to prove Theorem 1.1 and Theorem 1.2 it is enough to prove that

$$
|\{x:|\mathcal{T}| f|(x)|>2\}| \lesssim[W]_{\mathrm{A}_{\infty}^{\mathrm{sc}}}\|f\|_{L^{1}\left(\mathbb{R}^{d} ; \mathbb{C}^{n}\right)}
$$

where $\mathcal{T}$ is the scalar linear operator defined by

$$
\mathcal{T} h(x)=\sum_{Q \in \mathcal{S}}\left|W(x) \mathcal{W}_{Q}^{-1}\right|_{\mathrm{op}} f_{Q} h(y) d y \cdot \chi_{Q}(x)
$$

and $\mathcal{S}$ is a sparse family of cubes contained in a dyadic grid $\mathcal{D}$. Let

$$
\Omega=\left\{x: M^{\mathcal{D}} f(x)>2\right\}=\bigcup_{j} Q_{j}
$$

where $\left\{Q_{j}\right\}$ are the maximal dyadic cubes that satisfy:

$$
f_{Q_{j}}|f(y)| d y>2 .
$$

We use a Calderón-Zygmund decomposition argument inspired by the arguments in [5]. Let $|f|=g+b$ where $b=\sum_{j} b_{j}$ and $b_{j}=\left(|f|-f_{Q_{j}}|f|\right) \chi_{Q_{j}}$. Then $g$ is a non-negative function that satisfies

$$
g(x) \leq 2^{d+1} \quad \int_{\mathbb{R}^{d}} g(x) d x \leq \int_{\mathbb{R}^{d}}|f(x)| d x
$$

while each $b_{j}$ is supported on $Q_{j}$ and satisfies $\int_{Q_{j}} b_{j}(x) d x=0$. Then we have

$$
\begin{aligned}
|\{x:|\mathcal{T}| f|(x)|>2\}| \leq|\{x: \mathcal{T} g(x)>1\}| & \\
& +|\{x \in \Omega: \mathcal{T} b(x)>1\}|+|\{x \notin \Omega: \mathcal{T} b(x)>1\}| .
\end{aligned}
$$


Notice that the second term satisfies $|\Omega| \leq\|f\|_{L^{1}\left(\mathbb{R}^{d} ; \mathbb{C}^{n}\right)}$. Meanwhile, the third term is zero, since $\mathcal{T} b=0$. Indeed, if $x \in Q$ and $Q \subseteq Q_{j}$ for some $j$ then obviously $x \in \Omega$. Thus,

$$
\int_{Q} b(y) d y=\sum_{j: Q_{j} \subset Q} \int_{Q_{j}} b_{j}(y) d y=0
$$

so $\mathcal{T} b=0$. Thus we are reduced to estimating $|\{x: \mathcal{T} g(x)>1\}|$. By (2.1) and the sharp $\mathrm{A}_{\infty}$ reverse Hölder inequality (see [13]), we can pick $c>0$ independent of $W$ where if $q=1+\frac{c}{[W]_{\mathrm{A}_{\infty}^{\mathrm{sc}}}}$ then

$$
\begin{aligned}
f_{Q}\left|W(x) \mathcal{W}_{Q}^{-1}\right|_{\mathrm{op}}^{q} d x & \simeq \sum_{i=1}^{n} f_{Q}\left|W(x) \mathcal{W}_{Q}^{-1} e_{i}\right|^{q} d x \\
& \lesssim \sum_{i=1}^{n}\left(f_{Q}\left|W(x) \mathcal{W}_{Q}^{-1} e_{i}\right| d x\right)^{q} \simeq \sum_{i=1}^{n}\left|\mathcal{W}_{Q} \mathcal{W}_{Q}^{-1} e_{i}\right|^{q} \simeq 1
\end{aligned}
$$

Let $r<q$ be such that $r^{\prime}=2 q^{\prime}$. We will show that $\|\mathcal{T}\|_{L^{r}\left(\mathbb{R}^{d}\right) \rightarrow L^{r}\left(\mathbb{R}^{d}\right)} \lesssim[W]_{\mathrm{A}_{\infty}^{\text {sc }}}$ and thus

$$
|\{x: \mathcal{T} g(x)>1\}| \leq \int_{\mathbb{R}^{n}} \mathcal{T} g(x)^{r} d x \lesssim[W]_{\mathrm{A}_{\infty}^{r}}^{r} \int_{\mathbb{R}^{d}} g(x)^{r} d x \lesssim[W]_{\mathrm{A}_{\infty}^{\mathrm{sc}}} \int_{\mathbb{R}^{d}}|f(x)| d x
$$

where in the last line we used that $[W]_{\mathrm{A}_{\infty}^{\mathrm{sc}}}^{r} \leq[W]_{\mathrm{A}_{\infty}^{\mathrm{sc}}}^{1+\frac{c}{\left[W \mathrm{~A}_{\infty}^{\mathrm{sc}}\right.}} \lesssim[W]_{\mathrm{A}_{\infty}^{\mathrm{sc}}}$. To see that $\mathcal{T}$ is bounded on $L^{r}\left(\mathbb{R}^{d}\right)$ let $g$ and $h$ be non-negative scalar functions with $g \in L^{r}\left(\mathbb{R}^{d}\right)$ and $h \in L^{r^{\prime}}\left(\mathbb{R}^{d}\right)$. Then we use the well-known bound for the dyadic maximal function

$$
\left(\int_{\mathbb{R}^{d}}\left(M^{\mathcal{D}} g(x)\right)^{r} d x\right)^{\frac{1}{r}} \leq r^{\prime}\left(\int_{\mathbb{R}^{d}}|g(x)|^{r} d x\right)^{\frac{1}{r}} \lesssim[W]_{\mathrm{A}_{\infty}^{\mathrm{sc}}}\left(\int_{\mathbb{R}^{d}}|g(x)|^{r} d x\right)^{\frac{1}{r}} .
$$

We have

$$
\begin{aligned}
& \int_{\mathbb{R}^{d}} \mathcal{T} g(x) h(x) d x=\sum_{Q \in \mathcal{S}} f_{Q}\left|W(x) \mathcal{W}_{Q}^{-1}\right|_{\mathrm{op}} h(x) d x f_{Q} g(y) d y|Q| \\
& \leq \sum_{Q \in \mathcal{S}}\left(f_{Q}\left|W(x) \mathcal{W}_{Q}^{-1}\right|_{\mathrm{op}}^{q} d x\right)^{\frac{1}{q}}\left(f_{Q} h(x)^{q^{\prime}} d x\right)^{\frac{1}{q^{\prime}}} f_{Q} g(y) d y|Q| \\
& \lesssim \sum_{Q \in \mathcal{S}}\left(f_{Q} h(x)^{q^{\prime}} d x\right)^{\frac{1}{q^{\prime}}} f_{Q} g(y) d y|Q| \\
& \leq\left[\sum_{Q \in \mathcal{S}}\left(f_{Q} h(x)^{q^{\prime}} d x\right)^{\frac{r^{\prime}}{q^{\prime}}}|Q|\right]^{\frac{1}{r^{\prime}}}\left[\sum_{Q \in \mathcal{S}}\left(f_{Q} g(x) d x\right)^{r}|Q|\right]^{\frac{1}{r}} \\
& \lesssim\left[\sum_{Q \in \mathcal{S}}\left(f_{Q} h(x)^{q^{\prime}} d x\right)^{\frac{r^{\prime}}{q^{\prime}}}\left|E_{Q}\right|\right]^{\frac{1}{r^{\prime}}}\left[\sum_{Q \in \mathcal{S}}\left(f_{Q} g(x) d x\right)^{r}\left|E_{Q}\right|\right]^{\frac{1}{r}}
\end{aligned}
$$




$$
\begin{aligned}
& \leq\left[\int_{\mathbb{R}^{d}} M_{q^{\prime}}^{\mathcal{D}} h(x)^{r^{\prime}} d x\right]^{\frac{1}{r^{\prime}}}\left[\int_{\mathbb{R}^{d}} M^{\mathcal{D}} g(x)^{r} d x\right]^{\frac{1}{r}} \\
& \lesssim[W]_{\mathrm{A}_{\infty}^{\mathrm{sc}}}\|h\|_{L^{r^{\prime}\left(\mathbb{R}^{d}\right)}}\|g\|_{L^{r}\left(\mathbb{R}^{d}\right)}
\end{aligned}
$$

where we have additionally used that the maximal function $M_{q^{\prime}}^{\mathcal{D}} h=M\left(|h|^{q^{\prime}}\right)^{\frac{1}{q^{\prime}}}$ is bounded on $L^{r^{\prime}}\left(\mathbb{R}^{d}\right)$ since $q^{\prime}<r^{\prime}=2 q^{\prime}$ and

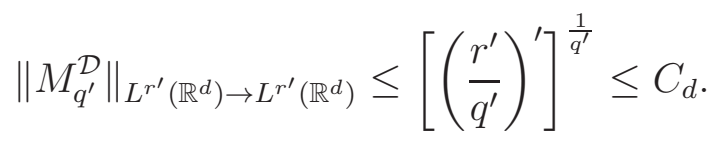

Remark. The proof of Theorem 1.1 using Lemma 3.1 has the advantage that it allows us to simultaneously prove Theorem 1.2. However, it is also possible to prove the endpoint estimate for $M_{W}$ ( most likely with worse $\mathrm{A}_{1}$ and $\mathrm{A}_{\infty}^{\mathrm{sc}}$ dependence) more directly by modifying the original proof of the strong $(p, p)$ estimates due to Goldberg [10]. This proof also relies on the Calderón-Zygmund decomposition into "good" and "bad" functions. The estimate for the bad part is similar to the proof given above, while the estimate for the good part is more complicated and relies on the operator $N_{Q}$ from [10]. We leave the details of this proof to the interested reader.

\section{Proof of Theorem 1.3}

The Calderón-Zygmund decomposition argument that was used to prove Theorem 1.2 unfortunately does not work to handle the sparse type operators in Theorem 2.3 and instead we need to employ "slicing" arguments that are similar to the ones in [1].

First, we recall a few facts about the space $L \log L$ and $\exp L$ needed in the proof. For details, see [4, Chapter 5]. Let $\Phi(t)=t \log (e+t)$. It is straightforward to show that $\Phi$ is submultiplicative: for all $s, t>0, \Phi(s t) \lesssim \Phi(s) \Phi(t)$. For a measurable, $\mathbb{C}^{n}$ valued function $f$ and a measurable $Q \subset \mathbb{R}^{d}$ with $0<|Q|<\infty$, define the $L \log L$ norm by the Luxemburg norm

$$
\||f|\|_{L \log L, Q}=\inf \left\{\lambda>0: f_{Q} \Phi\left(\frac{|f(y)|}{\lambda}\right) d y \leq 1\right\} .
$$

The conjugate Young function of $\Phi$ is the function $\bar{\Phi}(t)=e^{t}-1$. We again define the $\exp L$ norm by the Luxemburg norm

$$
\||f|\|_{\exp L, Q}=\inf \left\{\lambda>0: f_{Q} \bar{\Phi}\left(\frac{|f(y)|}{\lambda}\right) d y \leq 1\right\} .
$$

Then we have the following Hölder inequality for these spaces:

$$
f_{Q}|h(x) g(x)| d x \lesssim\|h\|_{L \log L, Q}\|g\|_{\exp L, Q} .
$$


Finally we will need the exponential integrability of $B M O$ functions; this is a consequence of the classical John-Nirenberg theorem. If $b \in B M O$, then

$$
\left\|b-b_{Q}\right\|_{\exp L, Q} \leq c\|b\|_{B M O} .
$$

We will prove the desired estimate in Theorem 1.3 by first bounding the term (2.2). This bound is given by the following lemma.

Lemma 4.1. Let $\mathcal{S}$ be a sparse family and

$$
\mathcal{T}_{\mathcal{S}, b, W} f(x)=\sum_{Q \in \mathcal{S}}\left|b(x)-b_{Q}\right|\left(f_{Q}\left|W(x) W^{-1}(y) f(y)\right| d y\right) \chi_{Q}(x) .
$$

If $b \in B M O$ then

$$
\left|\left\{\mathcal{T}_{\mathcal{S}, b, W} f(x)>\lambda\right\}\right| \lesssim \lambda^{-1}\|b\|_{B M O}[W]_{A_{1}}[W]_{A_{\infty}^{s c}} \max \left\{\log \left([W]_{A_{1}}+e\right),[W]_{A_{\infty}^{s c}}\right\}\|f\|_{L^{1}}
$$

Proof. Without loss of generality we may assume that $\lambda=1$ and $\|f\|_{L^{1}}=\|b\|_{B M O}=$ 1. Furthermore, we may assume that $\mathcal{S}$ is $\frac{4}{5}$ sparse. If

$$
G=\left\{\left|\mathcal{T}_{\mathcal{S}, b, W} f(x)\right|>1\right\} \backslash\{M(|f|)(x)>1\} .
$$

then it suffices to prove that

$$
|G| \leq c_{d}[W]_{A_{1}}[W]_{\mathrm{A}_{\infty}^{\mathrm{sc}}} \max \left\{\log \left([W]_{A_{1}}+e\right),[W]_{\mathrm{A}_{\infty}^{\mathrm{sc}}}\right\}+\frac{1}{2}|G| .
$$

Let $g=\chi_{G}$ and as before choose $s=1+\frac{c}{[W]_{\mathrm{A}_{\infty}^{\mathrm{sc}}}}$ with $c$ independent of $W$ where

$$
\left(f_{Q}\left|W(x) \mathcal{W}_{Q}^{-1}\right|_{\mathrm{op}}^{s} d x\right)^{\frac{1}{s}} \lesssim f_{Q}\left|W(x) \mathcal{W}_{Q}^{-1}\right|_{\mathrm{op}} d x \approx\left|\mathcal{W}_{Q} \mathcal{W}_{Q}^{-1}\right|_{\mathrm{op}}=1
$$

We then have

$$
|G|=\left|\left\{x \in G: \mathcal{T}_{\mathcal{S}, b, W} f(x)>1\right\}\right| \leq \int_{G} \mathcal{T}_{\mathcal{S}, b, W} f(x) d x
$$

while

$$
\begin{aligned}
\int_{G} \mathcal{T}_{\mathcal{S}, b, W} f(x) d x & \\
& =\sum_{Q \in \mathcal{S}} f_{Q} \int_{Q \cap G}\left|b(x)-b_{Q}\right|\left|W(x) W^{-1}(y) f(y)\right| d x d y \\
& \leq \sum_{Q \in \mathcal{S}} f_{Q} g(x)\left|b(x)-b_{Q}\right|\left|W(x) \mathcal{W}_{Q}^{-1}\right|_{\mathrm{op}} d x \int_{Q}\left|\mathcal{W}_{Q} W^{-1}(y) f(y)\right| d y \\
& \leq \sum_{Q \in \mathcal{S}}\left(f_{Q}\left|W(x) \mathcal{W}_{Q}^{-1}\right|_{\mathrm{op}}^{s} d x\right)^{\frac{1}{s}}\left(f_{Q} g\right)^{\frac{1}{2 s^{\prime}}}\left(f_{Q}\left|b(x)-b_{Q}\right|^{2 s^{\prime}} d x\right)^{\frac{1}{2 s^{\prime}}}
\end{aligned}
$$




$$
\begin{gathered}
\times \int_{Q}\left|\mathcal{W}_{Q} W^{-1}(y) f(y)\right| d y|Q| \\
\leq c_{d}[W]_{A_{1}}[W]_{\mathrm{A}_{\infty}^{\mathrm{sc}}} \sum_{Q \in \mathcal{S}}\left(f_{Q} g\right)^{\frac{1}{2 s^{\prime}}} f_{Q}|f(y)| d y|Q| .
\end{gathered}
$$

Here we have used the following corollary of John-Nirenberg inequality

$$
\left(f_{Q}\left|b(x)-b_{Q}\right|^{2 s^{\prime}} d x\right)^{\frac{1}{2 s^{\prime}}} \lesssim s^{\prime}\|b\|_{B M O} \approx[W]_{\mathrm{A}_{\infty}^{\mathrm{sc}}}
$$

see for instance [8, Corollary 3.10 p.166]. Now we split the sparse family as follows: We say $Q \in \mathcal{S}_{k, j}, k, j \geq 0$ if

$$
\begin{aligned}
& 2^{-j-1}<f_{Q}|f(y)| d y \leq 2^{-j}, \\
& 2^{-k-1}<\langle g\rangle_{Q}^{\frac{1}{2 s^{\prime}}} \leq 2^{-k}
\end{aligned}
$$

Then

$$
\begin{aligned}
\int_{G} \mathcal{T}_{\mathcal{S}, b, W} f(x) d x & \leq c_{d}[W]_{A_{1}}[W]_{\mathrm{A}_{\infty}^{\mathrm{sc}}} \sum_{j=0}^{\infty} \sum_{k=0}^{\infty} \sum_{Q \in \mathcal{S}_{k, j}}\left(f_{Q} g\right)^{\frac{1}{2 s^{\prime}}} f_{Q}|f(y)| d y|Q| \\
& =\sum_{k=0}^{\infty} \sum_{j=0}^{\infty} s_{k, j} .
\end{aligned}
$$

Now we observe that

$$
s_{k, j} \leq \min \left\{c_{d} 2 \cdot 2^{-k}[W]_{A_{1}}[W]_{\mathrm{A}_{\infty}^{\mathrm{sc}}}, c_{d} 2^{-j} 2^{k\left(2 s^{\prime}-1\right)+2 s^{\prime}}[W]_{A_{1}}[W]_{\mathrm{A}_{\infty}^{\mathrm{sc}}}|G|\right\}:=\alpha_{k, j} .
$$

For the first estimate we argue as follows. Let $E_{Q}=Q \backslash \bigcup_{Q^{\prime} \in \mathcal{S}_{j, k}} Q^{\prime}$. Then

$$
\begin{aligned}
\int_{Q}|f(y)| d y & =\int_{E_{Q}}|f(y)| d y+\int_{\bigcup_{Q^{\prime} \subsetneq Q}}|f(y)| d y \\
& \leq \int_{E_{Q}}|f(y)| d y+\sum_{Q^{\prime} \subsetneq Q} \int_{Q^{\prime}}|f(y)| d y .
\end{aligned}
$$

For the second term,

$$
\begin{aligned}
\sum_{Q^{\prime} \subsetneq Q} \int_{Q^{\prime}}|f(y)| d y & \leq 2^{-j} \sum_{Q^{\prime} \subsetneq Q, Q^{\prime} \in \mathcal{S}_{k, j}}\left|Q^{\prime}\right| \\
& \leq 2^{-j-2}|Q| \\
& \leq \frac{1}{2} \int_{Q}|f(y)| d y
\end{aligned}
$$


since $\mathcal{S}$ is $\frac{4}{5}$ sparse and thus $\frac{5}{4}$ Carleson. Thus,

$$
\int_{Q}|f(y)| d y \leq 2 \int_{E_{Q}}|f(y)| d y .
$$

which means that

$$
\begin{aligned}
s_{k, j} & \leq 2 \sum_{Q \in \mathcal{S}_{j, k}} \int_{E_{Q}}|f|\left(f_{Q} g\right)^{\frac{1}{2 s^{\prime}}} \\
& \leq 2 \cdot 2^{-k} \sum_{Q \in \mathcal{S}_{j, k}} \int_{E_{Q}}|f| \\
& \leq 2 \cdot 2^{-k} \int_{\mathbb{R}^{d}}|f|=2 \cdot 2^{-k} .
\end{aligned}
$$

For the second estimate of $s_{k, j}$, let $S_{j, k}^{*}$ denote the maximal cubes in $S_{j, k}$. Then

$$
\begin{aligned}
s_{k, j} & \leq 2^{-j} 2^{-k} \sum_{Q \in \mathcal{S}_{j, k}}|Q| \\
& \leq 2^{-j} 2^{-k} \sum_{Q \in \mathcal{S}_{j, k}^{*}} \sum_{P \subseteq Q}|P| \\
& \leq \frac{5}{4} 2^{-j} 2^{-k} \sum_{Q \in \mathcal{S}_{j, k}^{*}}|Q| \\
& =\frac{5}{4} 2^{-j} 2^{-k}\left|\bigcup_{Q \in \mathcal{S}_{j, k}} Q\right| \\
& =\frac{5}{4} 2^{-j} 2^{-k}\left|\left\{x \in \mathbb{R}^{d}: M g>2^{-2 s^{\prime} k-2 s^{\prime}}\right\}\right| \\
& \leq c_{d} 2^{-j} 2^{k\left(2 s^{\prime}-1\right)+2 s^{\prime}}|G| .
\end{aligned}
$$

Since $\mathcal{S}$ is $\frac{5}{4}$ Carleson. Putting all this together, we obtain

$$
|G| \leq \sum_{k=0}^{\infty} \sum_{j=0}^{\infty} \alpha_{k, j}
$$

Pick some $\gamma>0$ to be determined momentarily. To finish the proof we will estimate the double sum

$$
\begin{aligned}
\sum_{k=0}^{\infty} \sum_{j=0}^{\infty} \alpha_{k, j}= & \sum_{j \geq\left\lceil\log _{2}\left([W]_{A_{1}}[W]_{A_{\infty}} \gamma\right)\right\rceil+\left\lceil k\left(2 s^{\prime}-1\right)+2 s^{\prime}\right\rceil+k} \alpha_{k, j} \\
& +\sum_{j<\left\lceil\log _{2}\left([W]_{A_{1}}[W]_{A_{\infty}} \gamma\right)\right\rceil+\left\lceil k\left(2 s^{\prime}-1\right)+2 s^{\prime}\right\rceil+k} \alpha_{k, j} .
\end{aligned}
$$


For the first term

$$
\begin{aligned}
& \sum_{j \geq\left\lceil\log _{2}\left([W]_{A_{1}}[W]_{A_{\infty}} \gamma\right)\right\rceil+\left\lceil k\left(2 s^{\prime}-1\right)+2 s^{\prime}\right\rceil+k} \alpha_{k, j} \\
& \leq c_{d}[W]_{A_{1}}[W]_{\mathrm{A}_{\infty}^{\mathrm{sc}}}|G| \sum_{k=0}^{\infty} 2^{k\left(2 s^{\prime}-1\right)+2 s^{\prime}} \sum_{j \geq\left\lceil\log _{2}\left([W]_{A_{1}}[W]_{\mathrm{A}_{\infty}^{\mathrm{sc}} \gamma} \gamma\right)\right\rceil+\left\lceil k\left(2 s^{\prime}-1\right)+2 s^{\prime}\right\rceil+k} 2^{-j} \\
& =c_{d}[W]_{A_{1}}[W]_{\mathrm{A}_{\infty}^{\mathrm{sc}}}|G| \sum_{k=0}^{\infty} 2^{k\left(2 s^{\prime}-1\right)+2 s^{\prime}} 2^{-\left\lceil\log _{2}\left([W]_{A_{1}}[W]_{\mathrm{A}_{\infty}^{\mathrm{scc}} \gamma} \gamma\right)\right\rceil-\left\lceil k\left(2 s^{\prime}-1\right)+2 s^{\prime}\right\rceil-k} \\
& =c_{d}[W]_{A_{1}}[W]_{\mathrm{A}_{\infty}^{\mathrm{sc}}}|G| \sum_{k=0}^{\infty} 2^{k\left(2 s^{\prime}-1\right)+2 s^{\prime}} 2^{-\left\lceil\log _{2}\left([W]_{A_{1}}[W]_{\mathrm{A}_{\infty}^{\mathrm{scc}} \gamma} \gamma\right)\right\rceil-\left\lceil k\left(2 s^{\prime}-1\right)+2 s^{\prime}\right\rceil-k} \\
& \leq \frac{c_{d}[W]_{A_{1}}[W]_{\mathrm{A}_{\infty}^{\mathrm{sc}}}|G|}{\left[W \sum_{A_{1}}[W]_{\mathrm{A}_{\infty}^{\mathrm{sc}} \gamma}^{\infty} 2^{-k} \leq \frac{2 c_{d}}{\gamma}|G| .\right.}
\end{aligned}
$$

And it suffices to choose $\gamma=4 c_{d}$. For the second term

$$
\begin{aligned}
& \sum_{j<\left\lceil\log _{2}\left([W]_{A_{1}}[W]_{\mathrm{A}_{\infty}^{\mathrm{sc}} 4 c_{d}}\right)\right\rceil+k} \alpha_{k, j} \\
\leq & c_{d} 2 \cdot \sum_{k=0}^{\infty} \sum_{1 \leq j<\left[\log _{2}\left([W]_{A_{1}}[W]_{\mathrm{A}_{\infty}^{\mathrm{sc}} 4 c_{d}}\right)\right\rceil+\left\lceil k\left(2 s^{\prime}-1\right)+2 s^{\prime}\right\rceil+k} 2^{-k}[W]_{A_{1}}[W]_{\mathrm{A}_{\infty}^{\mathrm{sc}}} \\
\leq & c_{d} 2 \cdot \sum_{k=0}^{\infty}\left(\log _{2}\left([W]_{A_{1}}[W]_{\mathrm{A}_{\infty}^{\mathrm{sc}} 4} 4 c_{d}\right)+2(k+1) s^{\prime}\right) 2^{-k}[W]_{A_{1}}[W]_{\mathrm{A}_{\infty}^{\mathrm{sc}}} \\
\leq & c_{d}[W]_{A_{1}}[W]_{\mathrm{A}_{\infty}^{\mathrm{sc}}} \max \left\{\log \left([W]_{A_{1}}+e\right),[W]_{\mathrm{A}_{\infty}^{\mathrm{sc}}}\right\} .
\end{aligned}
$$

Combining all the preceding estimates we are done.

We now finish the proof of Theorem 1.3 by estimating (2.3). More precisely, standard bounds on $M_{\Phi}$ in conjunction with the following lemma will finish the proof of Theorem 1.3. To prove the following Lemma we will need the exponential integrability of $B M O$ functions and

Lemma 4.2. Let $\mathcal{S}$ be a $\frac{2 \Phi(2)}{2 \Phi(2)+1}$ sparse family and let

$$
\mathcal{T}_{S, b, W}^{*} f(x)=\sum_{Q \in \mathcal{S}} f_{Q}\left(\left|b(y)-b_{Q}\right|\left|W(x) W^{-1}(y) f(y)\right| d y\right) \chi_{Q}(x) .
$$

If $b \in B M O$ with $\|b\|_{B M O}=1$ and

$$
G=\left\{\left|\mathcal{T}_{b, W}^{*} f(x)\right|>1\right\} \backslash\left\{M_{\Phi}(|f|)(x)>1\right\}
$$


then we have that

$$
|G| \leq c_{d}[W]_{A_{1}} \max \left\{\log \left([W]_{A_{1}}+e\right),[W]_{A_{\infty}^{s c}}\right\}^{2} \int_{\mathbb{R}^{d}} \Phi(|f|)+\frac{1}{2}|G| .
$$

Proof. Since $b \in B M O$ with $\|b\|_{B M O}=1$, by (4.3) there exists a constant $c_{d}$ such that

$$
\left\|b-b_{Q}\right\|_{\exp L, Q} \leq c_{d}
$$

If we again denote $g=\chi_{G}$ and proceed as in the proof of Lemma 4.1 then

$$
\begin{aligned}
& \sum_{Q \in \mathcal{S}} f_{Q} \int_{Q \cap G}\left|b(y)-b_{Q}\right|\left|W(x) W^{-1}(y) f(y)\right| d x d y \\
& \leq \sum_{Q \in \mathcal{S}} f_{Q}\left|W(x) \mathcal{W}_{Q}^{-1}\right|_{\mathrm{op}} g(x) d x f_{Q}\left|b(y)-b_{Q}\right|\left|\mathcal{W}_{Q} W^{-1}(y) f(y)\right| d y|Q| \\
& \leq \sum_{Q \in \mathcal{S}}\left(f_{Q}\left|W(x) \mathcal{W}_{Q}^{-1}\right|_{\mathrm{op}}^{s} d x\right)^{\frac{1}{s}}\left(f_{Q} g\right)^{\frac{1}{s^{\prime}}} f_{Q}\left|b(y)-b_{Q}\right|\left|\mathcal{W}_{Q} W^{-1}(y) f(y)\right| d y|Q| \\
& \leq \sum_{Q \in \mathcal{S}}\left(f_{Q}\left|W(x) \mathcal{W}_{Q}^{-1}\right|_{\mathrm{op}}^{s} d x\right)^{\frac{1}{s}}[W]_{A_{1}}\left(f_{Q} g\right)^{\frac{1}{s^{\prime}}} f_{Q}\left|b(y)-b_{Q}\right||f(y)| d y|Q| \\
& \leq c_{d}[W]_{A_{1}} \sum_{Q \in \mathcal{S}} f_{Q}\left|W(x) \mathcal{W}_{Q}^{-1}\right|_{\mathrm{op}} d x\left(f_{Q} g\right)^{\frac{1}{s^{\prime}}}\left\|b-b_{Q}\right\|_{\exp L, Q}|||f| \|_{L \log L, Q}|Q| \\
& \leq c_{d}[W]_{A_{1}} \sum_{Q \in \mathcal{S}}\left(f_{Q} g\right)^{\frac{1}{s^{\prime}}}\||f|\|_{L \log L, Q}|Q| .
\end{aligned}
$$

As before we say $Q \in \mathcal{S}_{k, j}$ if

$$
\begin{aligned}
& 2^{-j-1}<\|\mid f\|_{L \log L, Q} \leq 2^{-j} \\
& 2^{-k-1}<\langle g\rangle_{Q}^{\frac{1}{s^{\prime}}} \leq 2^{-k}
\end{aligned}
$$

Then

$$
\begin{aligned}
& \sum_{Q \in \mathcal{S}}\left(f_{Q} g\right)^{\frac{1}{s^{\prime}}}\||f|\|_{L \log L, Q}|Q| \\
& =\sum_{j=0}^{\infty} \sum_{k=0}^{\infty} \sum_{Q \in \mathcal{S}_{k, j}}\left(f_{Q} g\right)^{\frac{1}{s^{\prime}}}\||f|\|_{L \log L, Q}|Q| \\
& =\sum_{k=0}^{\infty} \sum_{j=0}^{\infty} s_{k, j} .
\end{aligned}
$$


Now we are going to prove that

$$
s_{k, j} \leq \min \left\{c_{d} 2^{-k} j \int_{\mathbb{R}^{d}} \Phi(|f(y)|) d y, c_{d} 2^{-j} 2^{k\left(s^{\prime}-1\right)+s^{\prime}}|G|\right\}:=\alpha_{k, j} .
$$

We start with the first estimate. Again let $E_{Q}=Q \backslash \bigcup_{\substack{Q^{\prime} \in \mathcal{S}_{k, j} \\ Q^{\prime} \subseteq Q}} Q^{\prime}$. First we note that

$$
\int_{Q} \Phi\left(2^{j}|f(y)|\right) d y \leq 2 \int_{E_{Q}} \Phi\left(2^{j}|f(y)|\right) d y .
$$

Indeed, since $\mathcal{S}_{k, j}$ is $1+\frac{1}{2 \Phi(2)}$-Carleson and $2^{-j-1}<\||f|\|_{L \log L, Q} \leq 2^{-j}$ we have that

$$
\begin{aligned}
& \int_{Q} \Phi\left(2^{j}|f(y)|\right) d y \\
& \leq \int_{E_{Q}} \Phi\left(2^{j}|f(y)|\right) d y+\sum_{Q^{\prime} \subsetneq Q, Q^{\prime} \in \mathcal{S}_{k, j}} \int_{Q^{\prime}} \Phi\left(2^{j}|f(y)|\right) d y \\
& \leq \int_{E_{Q}} \Phi\left(2^{j}|f(y)|\right) d y+\sum_{Q^{\prime} \subsetneq Q, Q^{\prime} \in \mathcal{S}_{k, j}}\left|Q^{\prime}\right| \\
& \leq \int_{E_{Q}} \Phi\left(2^{j}|f(y)|\right) d y+\frac{1}{2 \Phi(2)} \int_{Q} \Phi\left(2^{j+1}|f(y)|\right) d y \\
& \leq \int_{E_{Q}} \Phi\left(2^{j}|f(y)|\right) d y+\frac{1}{2} \int_{Q} \Phi\left(2^{j}|f(y)|\right) d y .
\end{aligned}
$$

Now we observe that

$$
\begin{aligned}
s_{k, j} & \leq 2^{-j} \sum_{Q \in \mathcal{S}_{j, k}} \int_{Q} \Phi\left(2^{j+1}|f(y)|\right) d y\left(f_{Q} g\right)^{\frac{1}{s^{\prime}}} \\
& \leq c_{d} 2 \cdot 2^{-k} 2^{-j} \Phi(2) \sum_{Q \in \mathcal{S}_{j, k}} \int_{E_{Q}} \Phi\left(2^{j}|f(y)|\right) d y \\
& \leq c_{d} 2^{-k} \Phi(2) j \int_{\mathbb{R}^{d}} \Phi(|f(y)|) d y
\end{aligned}
$$

while the other estimate for $s_{j, k}$ follows from (4.4). Combining the preceding estimates we have that

$$
\begin{aligned}
|G| & \leq c_{d}[W]_{A_{1}} \sum_{k=0}^{\infty} \sum_{j=0}^{\infty} s_{k, j} \\
& \leq \sum_{k=0}^{\infty} \sum_{j=0}^{\infty} \min \left\{[W]_{A_{1}} c_{d} 2^{-k} j \int_{\mathbb{R}^{d}} \Phi(|f(y)|) d y, c_{d} 2^{-j} 2^{k\left(s^{\prime}-1\right)+s^{\prime}}[W]_{A_{1}}|G|\right\} \\
& =\sum_{k=0}^{\infty} \sum_{j=0}^{\infty} \alpha_{k, j}
\end{aligned}
$$


Now we are left with estimating the double sum. We proceed as follows.

$$
\sum_{k=0}^{\infty} \sum_{j=0}^{\infty} \alpha_{k, j}=\sum_{j \geq\left\lceil\log _{2}\left([W]_{A_{1}} \gamma\right)\right\rceil+\left\lceil k\left(s^{\prime}-1\right)+s^{\prime}\right\rceil+k} \alpha_{k, j}+\sum_{j<\left\lceil\log _{2}\left([W]_{A_{1}} \gamma\right)\right\rceil+\left\lceil k\left(s^{\prime}-1\right)+s^{\prime}\right\rceil+k} \alpha_{k, j}
$$

For the first term

$$
\begin{aligned}
& \sum_{j \geq\left\lceil\log _{2}\left([W]_{A_{1}} \gamma\right)\right\rceil+\left\lceil k\left(s^{\prime}-1\right)+s^{\prime}\right\rceil+k} \alpha_{k, j} \\
& \leq c_{d}[W]_{A_{1}}|G| \sum_{k=0}^{\infty} 2^{k\left(s^{\prime}-1\right)+s^{\prime}} \sum_{j \geq\left\lceil\log _{2}\left([W]_{A_{1}} \gamma\right)\right\rceil+\left\lceil k\left(s^{\prime}-1\right)+s^{\prime}\right\rceil+k} 2^{-j} \\
& =c_{d}[W]_{A_{1}}|G| \sum_{k=0}^{\infty} 2^{k\left(s^{\prime}-1\right)+s^{\prime}} 2^{-\left\lceil\log _{2}\left([W]_{A_{1}} \gamma\right)\right\rceil-\left\lceil k\left(s^{\prime}-1\right)+s^{\prime}\right\rceil-k} \\
& =c_{d}[W]_{A_{1}}|G| \sum_{k=0}^{\infty} 2^{k\left(s^{\prime}-1\right)+s^{\prime}} 2^{-\left\lceil\log _{2}\left([W]_{A_{1}} \gamma\right)\right\rceil-\left\lceil k\left(s^{\prime}-1\right)+s^{\prime}\right\rceil-k} \\
& \leq \frac{c_{d}[W]_{A_{1}}}{[W]_{A_{1}} \gamma}|G| \sum_{k=0}^{\infty} 2^{-k} \\
& \leq \frac{2 c_{d}}{\gamma}|G|=\frac{1}{2}|G|
\end{aligned}
$$

where for the last equality we choose $\gamma=4 c_{d}$. For the second term

$$
\begin{aligned}
& \sum_{j<\left\lceil\log _{2}\left([W]_{A_{1}} \gamma\right)\right\rceil+\left\lceil k\left(s^{\prime}-1\right)+s^{\prime}\right\rceil+k} \alpha_{k, j} \\
& \leq c_{d} 2[W]_{A_{1}} \int_{\mathbb{R}^{d}} \Phi(|f(y)|) d y \sum_{k=0}^{\infty} 2^{-k} \sum_{1 \leq j<\left\lceil\log _{2}\left([W]_{A_{1}} \gamma\right)\right\rceil+\left\lceil k\left(s^{\prime}-1\right)+s^{\prime}\right\rceil+k}^{j} \\
& \leq c_{d} 2[W]_{A_{1}} \int_{\mathbb{R}^{d}} \Phi(|f(y)|) d y \sum_{k=0}^{\infty} 2^{-k}\left(\left\lceil\log _{2}\left([W]_{A_{1}} \gamma\right)\right\rceil+\left\lceil k\left(s^{\prime}-1\right)+s^{\prime}\right\rceil+k\right)^{2} \\
& \leq c_{d} 2[W]_{A_{1}} \max \left\{\log \left([W]_{A_{1}}+e\right),[W]_{\mathrm{A}_{\infty}^{\mathrm{sc}}}\right\}^{2} \int_{\mathbb{R}^{d}} \Phi(|f(y)|) d y .
\end{aligned}
$$

\section{REFERENCES}

[1] M. Caldarelli and I. P. Rivera-Ríos, A sparse approach to mixed weak type inequalities, preprint (2018). arXiv:1812.08023. 
[2] M. Christ and M. Goldberg, Vector $A_{2}$ weights and a Hardy-Littlewood maximal function, Trans. Amer. Math. Soc. 353 (2001), 1995 -2002.

[3] D. Cruz-Uribe, J. Isralowitz, and K. Moen, Two weight bump conditions for matrix weights, Integr. Equ. Oper. Theory 90 (2018), no. 3.

[4] D. Cruz-Uribe, J. M. Martell, and C. Pérez, Weights, extrapolation and the theory of Rubio de Francia, Operator Theory: Advances and Applications, vol. 215, Birkhäuser/Springer Basel AG, Basel, 2011. MR2797562 (2012f:42001)

[5] D. Cruz-Uribe, J.M. Martell, and C. Pèrez, Weighted weak-type inequalities and a conjecture of sawyer, Int. Math. Res. Not. 30 (2005), $1849-1871$.

[6] D. Cruz-Uribe, K. Moen, and S. Rodney, Matrix $A_{p}$ weights, degenerate Sobolev spaces, and mappings of finite distortion, to appear, J. Geom. Anal.

[7] M. Frazier and S. Roudenko, Matrix-weighted Besov spaces and conditions of $A_{p}$ type for $0<$ $p \leq 1$., Indiana Univ. Math. J. 53 (2004), no. 5, 1225 -1254.

[8] José García-Cuerva and José L. Rubio de Francia, Weighted norm inequalities and related topics, North-Holland Mathematics Studies, vol. 116, North-Holland Publishing Co., Amsterdam, 1985. Notas de Matemática [Mathematical Notes], 104. MR807149

[9] T. A. Gillespie, S. Pott, S. Treil, and A. Volberg, Logarithmic growth for matrix martingale transforms., J. London Math. Soc. 642 (2001), no. 2, 624 -636.

[10] M. Goldberg, Matrix $A_{p}$ weights via maximal functions., Pacific J. Math. 211 (2003), no. 2, $201-220$.

[11] T. Hytönen, Dyadic analysis and weights, Lecture notes - Course at University of Helsinki, (2017). Available at: http://wiki.helsinki.fi/download/attachments/213996485/dyadic.pdf?api=v2.

[12] T. Hytönen and C. Pérez, Sharp weighted bounds involving $A_{\infty}$, Anal. PDE 6 (2013), no. 4, 777-818. MR3092729

[13] T. Hytönen, C. Pérez, and E. Rela, Sharp reverse Hölder property for $A_{\infty}$ weights on spaces of homogeneous type, J. Funct. Anal. 263 (2012), no. 12, 3883-3899. MR2990061

[14] J. Isralowitz and K. Moen, Matrix weighted Poincaré inequalities and applications to degenerate elliptic systems., Indiana Univ. Math. J. (to appear).

[15] J. Isralowitz, S. Pott, and I. P. Rivera-Ríos, Sharp $A_{1}$ weighted estimates for vector valued operators, preprint.

[16] A. Lerner, On pointwise estimates involving sparse operators, New York J. Math. 22 (2016), 341-349. MR3484688

[17] A. Lerner and F. Nazarov, Intuitive dyadic calculus: the basics, preprint (2015). arXiv:1508.05639.

[18] A. Lerner, F. Nazarov, and S. Ombrosi, On the sharp upper bound related to the weak muckenhoupt-wheeden conjecture., preprint (2018). arXiv:1710.07700.

[19] A. Lerner, S. Ombrosi, and C. Pérez, $A_{1}$ bounds for Calderón-Zygmund operators related to a problem of muckenhoupt and wheeden, Math. Res. Lett. 6 (2009), no. 16, 149 -156.

[20] A. Lerner, S. Ombrosi, and I.P. Rivera-Ríos, On pointwise and weighted estimates for commutators of Calderón-Zygmund operators, Adv. Math. 319 (2017), 153-181. MR3695871

[21] B. Muckenhoupt and R. Wheeden, Some weighted weak-type inequalities for the HardyLittlewood maximal function and the Hilbert transform, Indiana Univ. Math. J. 26 (1977), no. 5, 801-816. MR0447956

[22] F. Nazarov, S. Petermichl, S. Treil, and A. Volberg, Convex body domination and weighted estimates with matrix weights., Adv. Math. 318 (2017), 279 -306. 
[23] F. Nazarov and S. Treil, The hunt for a Bellman function: applications to estimates for singular integral operators and to other classical problems of harmonic analysis, Algebra i Analiz 8 (2017), no. 5, 32-162.

[24] C. Pérez, Endpoint estimates for commutators of singular integral operators, J. Funct. Anal. 128 (1995), no. 1, 163-185. MR1317714 (95j:42011)

[25] C. Pérez and G. Pradolini, Sharp weighted endpoint estimates for commutators of singular integrals, Michigan Math. J. 49 (2001), no. 1, 23-37. MR1827073

[26] S. Roudenko, Matrix-weighted Besov spaces, Trans. Amer. Math. Soc. 355 (2003), no. 1, 273314 (electronic). MR1928089 (2003k:42037)

[27] E. M. Stein and G. Weiss, Interpolation of operators with change of measures, Trans. Amer. Math. Soc. 87 (1958), 159-172. MR0092943 (19,1184d)

Department of Mathematics, University of Alabama, Box 870350, 345 Gordon Palmer Hall.

E-mail address: dcruzuribe@ua.edu

Department of Mathematics and Statistics, SUNY Albany, 1400 Washington Ave., Albany, NY, 12222.

E-mail address: jisralowitz@albany.edu

Department of Mathematics, University of Alabama, Box 870350, 345 Gordon PALMER Hall.

E-mail address: kabe.moen@ua.edu

Centre for Mathematical Sciences, University of Lund, PO Box 118, 22100 Lund, SWEDEN

E-mail address: sandra@maths.1th.edu

Instituto de Matemática de Bahía Blanca (INMABB), Departamento de Matemática, Universidad Nacional del Sur (UNS) - COniCET, Av. Alem 1253, Bahía Blanca, ArGENTINA

E-mail address: israel.rivera@uns.edu.ar 